\title{
Biopharmaceuticals: An emerging trend in Drug Development
}

\author{
Prashansa Agrawal* \\ Department of Chemistry, Case Western Reserve University, Cleveland, Ohio, 44106, USA
}

Received: December 09, 2014, Accepted: February 02, 2015, Published: February 06, 2015

*Corresponding author: Prashansa Agrawal, Department of Chemistry, Case Western Reserve University, Cleveland, Ohio, 44106, USA, Tel: +1 216368-2404; E-mail: prashansa.agrawal@case.edu

In the last two decades, the field of new drug development has moved its focus from small-molecule compounds to large-molecule proteins and other biopharmaceuticals. These biopharmaceuticals have caused an archetype shift in disease cure and has led to an enhancement in the quality of life of patients with various diseases such as autoimmune diseases, refractory cancers. In 1982, first biopharmaceutical product was approved by USFDA and since then the number has been increasing rapidly [1]. The recombinant protein therapeutic, Humulin, was introduced as biopharmaceutical in 1982. This was followed by therapeutic monoclonal antibody like Proleukin, and Zenapax (humanized monoclonal antibody) in 1990s. In 2003, Phage-evolved antibody, Humira was introduced by Egea Biosciences Inc.

At present, there has been a rapid advancement in the number of biopharmaceuticals available for clinical use. The United States Food and Drug Administration (USFDA) has approved 18 new biopharmaceutical products in 2012, which include eight recombinant proteins, along with two monoclonal antibodies and one engineered antibody-like biomolecule, covering a wide range of innovation, novelty, healthcare and market impact [1]. Research data released by PharmaVision in 2009 [2] shows that biopharmaceuticals represent $7.5 \%$ of all drugs in the market and account for $\sim 10 \%$ of the total expenses for marketed drugs. The usage of biopharmaceuticals are increasing at the rate $>20 \%$ per year. They are already being used $74 \%$ more than chemicallyderived pharmaceuticals, in life-saving or end-stage applications [2]. Moreover, biopharmaceuticals covers $>30 \%$ of all pipeline research programs going-on at present [2].

Biopharmaceuticals are defined in various different ways based on the opinion from different sources. In broad biotechnology term, biopharmaceuticals are defined as pharmaceuticals inherently biological in nature, extracted or manufactured by biotechnological methods, generally containing live entities or their active components [3]. This broad view has been implemented in United States and European Markets, which embraces all recombinant proteins, monoclonal antibodies, cytokines, peptides, vaccines, blood/plasma-derived products, non-recombinant culture-derived proteins, nucleic acid-based products (DNA, RNA or antisense oligonucleotides used for curative or in vivo diagnostic purposes), cells and tissues cultures. This view is possibly best understood by the public and mostly used by the people in the biopharmaceutical industry within the United States of America [4].

Second view, which is more prevalent in Europe, and/or is used by the people whose primary interest is in monoclonal antibody and recombinant products [4]. This view defines biopharmaceuticals as only those products, which consist of monoclonal antibodies and recombinant proteins as being biopharmaceuticals, excluding the non-recombinant cultured proteins, vaccines, blood proteins, plasma proteins and other classes of products. These are based on technologies developed in recent decades, like gene therapy based technique.

Third view is company-centric view and used by people in the press/media, stock analysts and industrial organization. By this view, products, technologies and companies do not need any contribution or usage of biotechnology to be named biopharmaceutical. This view includes everything as being biopharmaceutical from biotechnology-based smaller, commercial, R\&D-intensive, life science companies, pharmaceutical companies and biopharmaceuticals from large companies [5].

Fourth view is based on Pharma Business - This view includes biopharmaceuticals as a synonym for pharmaceuticals; and now consider the pharmaceutical industry as biopharmaceutical industry [5] due to the vital change experienced by the pharmaceutical industry, through implementation of new techniques and close interactions (predominantly outsourcing, contracting and licensing) with biotech companies. Various industrial analytical reports, together with those financed and delivered by the Pharmaceutical Research and Manufacturers Association, affirm that the pharmaceutical industries have just recently transformed into the biopharmaceutical industries as a consequence of the amalgamation of biotechnology and pharmaceutical industries.

In spite of above-mentioned controversies in the definition of biopharmaceuticals, the large variety of biopharmaceutical 
products are therapeutics that are extracted/produced from living organism. Recombinant human insulin was the first biopharmaceutical product approved for therapeutic purpose. Although biopharmaceuticals have high therapeutic potency, they have common glitches such as unanticipated side-effects and reduced pharmacological effect after repeated administration. Recently, a large number of disease-related proteins have been identified by genome or proteome analysis. However, there is still a limitation on the discovery of useful biomarkers for drug development due to the problem of efficiently selecting potential biomarkers from among many candidate proteins. Besides this, there are other complications that are needed to be considered. For example, biopharmaceuticals are difficult to manufacture and require specialized as well as expensive processing and purification methods. Their sensitivity to environmental conditions also makes hard to develop their biosimilars as a minor alteration in the production process may considerably disturb the safety and efficacy of the final product, therefore a control switch over every feature of the production process is crucial for the manufacturers of biopharmaceuticals. The technical hitches relating to the production is partially the reason for the availability of so few biosimilars as it is tremendously hard to reproduce an inventor's manufacturing process and produce an equivalent product.

The technology for biopharmaceutical development is not well established yet. New approaches and technologies need to be developed in order to overcome these problems. Establishment of a novel drug delivery system is required to improve in vivo stability and target selectivity of biopharmaceuticals. Efforts are required to integrate multiple approaches to create effective and safe biopharmaceuticals for their application to various diseases. Considering the constructive side, steps have been taken for the advancement of biopharmaceutical field, such as the development of disposable systems in bioprocessing that can reduce costs and compliance issues, setting up the specialist that can help in scaling up of commercial production in the companies. However, it needs more improvements as there will be an emerging need for more flexible production processes and facilities. This is mainly because of biopharmaceutical drugs become more specialized and directed to more focus and smaller market, which will require smaller consignment sizes.

It is obvious that biopharmaceuticals are going to be very crucial for the future growth of the pharmaceutical and biotechnology industry. Their market value is rising annually at the rate of $\sim 15 \%$ in comparison to only $\sim 6-7 \%$ for other pharmaceuticals $[6,7]$. In the next few years, there will be many biopharmaceuticals involving from novel classes of products: e.g., gene, stem cell and other cellular therapies, cancer vaccines, RNA interference, cultured tissue transplants, which are going to be great assets for the public health.

\section{References}

1. Rader RA. FDA Biopharmaceutical Product Approvals and Trends in 2012. BioProcess International. 2013; 11(3): 18-27.

2. Delivering New Biopharmaceutical Therapies: Challenges \& Opportunities. PharmaVision; [Published 2009 Jan 9]. Available from: http://www.pharmavision.co.uk/researchpapers/delivering+new+biophar maceutical+therapies $\% 3 \mathrm{~A}+$ challenges $+\% 26+$ opportunities- -32 .

3. Rader RA. Redefining biopharmaceutical. Nat Biotechnol. 2008; 26 (7): 743-751. doi: 10.1038/nbt0708-743.

4. Rader RA. What is a Biopharmaceutical? Part 1: (Bio) Technology-Based Definitions. BioExecutive International. 2005; 60-65.

5. Rader RA. What is a Biopharmaceutical? Part 2: Company and Industry Definitions. BioExecutive International. 2005; 42-49.

6. evaluategroup.com [Internet]. World Preview 2014, Outlook to 2020; [2014 June]. Available from: http://info.evaluategroup.com/rs/ evaluatepharmaltd/images/EP240614.pdf.

7. Pfizer [Internet]. Pfizer to Acquire Wyeth, Creating the World's Premier Biopharmaceutical Company c2009 [updated 2009 Jan 26]. Available from: http://press.pfizer.com/press-release/pfizer-acquirewyeth-creating-worlds-premier-biopharmaceutical-company. 